Giornale

\title{
Valutazione dello stato nutrizionale in pazienti emodializzati: ruolo dell'analisi bioimpedenziometrica
}

\author{
M.G. Chiappini, T. Ammann, D. Monno, G. Selvaggi, P. Traietti \\ Servizio di Nefrologia ed Emodialisi, AFaR-CRCCS, Ospedale Fatebenefratelli, \\ Isola Tiberina, Roma
}

A

lterazioni dello stato nutrizionale sono presenti in una elevata percentuale di pazienti con insufficienza renale cronica (IRC) in trattamento emodialitico periodico $(1,2)$. Nei numerosi studi effettuati sull'argomento la prevalenza di malnutrizione viene registrata anche fino al 40-70\% (3). Molteplici sono le cause di malnutrizione in questi soggetti: alterazioni del metabolismo calorico e proteico, disordini ormonali, malattie acute intercorrenti, condizioni cliniche associate all'IRC a loro volta causa di malnutrizione, stato ipercatabolico, persistenza di anoressia, nausea, vomito legati alla stessa tossicità uremica (4).

Diversi studi hanno dimostrato il possibile ruolo della malnutrizione nell'aumento della morbilità e mortalità dei pazienti dializati (5-7). Non si può escludere, peraltro, che le stesse frequenti complicanze acute intercorrenti e le numerose malatttie croniche associate possano esse stesse essere causa di malnutrizione influenzando di per sé negativamente la prognosi di questi soggetti.

I metodi di studio dello stato nutrizionale proposti nei pazienti in trattamento dialitico sono diversi. I più comuni e frequentemente utilizzati sono: valutazione dell' apporto alimen- tare ottenuto mediante inchiesta dietetica; il "subjective global assessment"; parametri antropometrici, bioumorali e immunitari. L'esame impedenziometrico (BIA), ampiamente utilizzato per lo studio del contenuto idrico dell'organismo, è stato introdotto negli ultimi anni quale valido metodo per lo studio della composizione corporea (8-10). Resta tuttavia ancora oggetto di studio quale sia il ruolo effettivo del BIA nella valutazione dello stato nutrizionale dei pazienti emodializati e quali parametri derivati da questo esame possano essere considerati i più attendibili nell'indicare la composizione corporea di questi soggetti.

Scopo del presente studio è: confrontare la valutazione dello stato nutrizionale ottenuta mediante esame nutrizionale "standard" ed esame BIA; valutare il valore clinico e prognostico degli indici impedenziometrici.

\section{Pazienti e metodi}

Sono stati studiati 134 pazienti (51 femmine, 83 maschi) di età media 62 \pm 14 anni (estremi 25-84) con IRC in trattamento emodialitico periodico da almeno 6 mesi (età dialitica media 62 \pm 45 mesi, estremi 6-243). Tutti i pazienti erano in condizioni cliniche stabili. 87 dei 134 pazienti studiati sono stati seguiti per 48 mesi.

Lo stato nutrizionale è stato valutato mediante esame "standard" ed esame BIA.

Per l'esame "standard" sono stati considerati i seguenti parametri:

- indici antropometrici (espressi in \% rispetto al valore standard): Body Mass Index (BMI), peso corporeo ideale (IBW), plica tricipitale (TST), circonferenza del muscolo del braccio (MAMC), grasso corporeo totale (\%BF);

- indici bioumorali (espressi in \% rispetto al valore normale): albumina (ALB), prealbumina (PRE), transferrina (TRF), C3;

- indici immunitari (espressi in \% rispetto al valore normale): conta dei linfociti totali (TLC), multitest (MTMerieux).

Per la valutazione dei parametri antropometrici sono stati considerati il peso corporeo, l'altezza, la circonferenza del polso, la circonferenza del braccio e la misura di quattro pliche cutanee (tricipitale, bicipitale, sottoscapolare, soprailiaca) ottenuta mediante plicometro di Harpender. Le misure antropometriche sono state rilevate sempre dallo stesso operatore al termine della seduta emodialitica, 
TABELLA I - CORRELAZIONI SIGNIFICATIVE

\begin{tabular}{lrrrrr}
\hline & AGE & AI & VPCI & II & NI \\
\hline AGE & - & -.23 & -.22 & - & -.30 \\
Rc & - & -.21 & -.27 & - & -.27 \\
XC & -.61 & - & .23 & .28 & .27 \\
PA & -.66 & .39 & .37 & .40 & .50 \\
BCM & -.62 & - & .33 & .30 & .21 \\
EC/BC & .62 & -.28 & -.39 & -.40 & -.47 \\
TBW & - & -.63 & - & - & -.39 \\
ECW & .49 & -.20 & -.31 & -.20 & -.31 \\
FM & - & .57 & - & - & 28 \\
FFM & - & -.55 & - & - & -.29 \\
\hline$r>17=p<.05 ;$ & & $r>.22=p<.01 ;$ & & $r>.28=p<.001$ &
\end{tabular}

dal lato controlaterale alla fistola arterovenosa. BMI è stato calcolato dividendo il peso corporeo per l'alteza al quadrato. MAMC è stata ottenuta dalla circonferenza del braccio (MAC) e dalla plica tricipitale secondo la seguente formula:

MAMC $=$ MAC $(\mathrm{cm})-0.314 \times$ TST (mm)

$\% \mathrm{BF}$ è stato calcolato secondo l'equazione:

$$
\% \mathrm{BF}=\left(\frac{4.95}{\text { densità corporea }}-4.50\right) \times 100
$$

dove la densità corporea deriva dalla somma delle quattro pliche cutanee secondo le tavole di Durnin (11).

Da questi parametri nutrizionali sono stati calcolati una serie di indici "sintetici" utilizzati per ottenere una valutazione semplificata dello stato nutrizionale mediante un numero più limitato di indici:

Indice Antropometrico $(\mathrm{AI})=(\mathrm{TST}$ + MAMC) $/ 2$;

Indice delle proteine viscerali (VP$\mathrm{CI})=(\mathrm{ALB}+\mathrm{PRE}+\mathrm{TRF}+\mathrm{C} 3) / 4$;

Indice immunitario (II) $=(\mathrm{TLC}+$ MT)/2;

Indice Nutrizionale $(\mathrm{NI})=(\mathrm{AI}+\mathrm{VP}-$ $\mathrm{CI}+\mathrm{II}) / 3$.

Viene considerato normale un valore di AI, VPCI, II e NI > 85\%.

L'esame BIA (ottenuto mediante BIA 101, Akern RJL System, Firenze) è stato effettuato secondo metodi- ca standard mezz'ora dopo il termine della seduta emodialitica. Dall'esame BIA sono stati ottenuti i seguenti indici misurati direttamente o calcolati mediante software:

resistenza ( $\mathrm{Rc})$, reattanza $(\mathrm{Xc})$, angolo di fase (PA), massa cellulare $(\mathrm{BCM} \%)$, massa grassa (FM\%), massa priva di grasso (FFM\%), acqua corporea totale (TBW\%), acqua extracellulare (ECW\%), massa extracellulare/massa cellulare (ECM/ BCM).

\section{Risultati}

Sulla base dell'esame nutrizionale "standard" la prevalenza di malnutrizione $(\mathrm{NI}<85 \%)$ è risultata pari al $41 \%$. Con la stessa distribuzione percentuale tra i maschi $(41 \%)$ e le femmine $(41 \%)$. Considerando soltanto AI quale indice di composizione corporea ottenuto mediante plicometria, questo risultava inferiore a $85 \%$ in una percentuale più bassa di pazienti pari al 33\% (49\% nei maschi e $23 \%$ nelle femmine).

Nelle Figure 1, 2 e 3 sono riportati i parametri derivati dal BIA rispettivamente nei pazienti considerati normali e malnutriti secondo l'esame nutrizionale "standard". Rc risultava significativamente più alta e Xc più bassa nei pazienti normali. Tale differenza non veniva registrata considerando soltanto AI. PA era signifi- cativamente più alto nei soggetti con normale stato nutrizionale. TBW, ECW e ECM/BCM erano significativamente più alti nei pazienti malnutriti ad indicare, secondo una prima interpretazione dei dati, uno stato di iperidratazione di questi soggetti. FM era significativamente più alta nei pazienti con normale AI mentre non è stata registrata alcuna differenza di questo parametro tra i pazienti suddivisi sulla base della valutazione globale dello stato nutrizionale. FFM risultava paradossalmente più alta nei pazienti considerati malnutriti sia sulla base di NI che di AI. $\mathrm{BCM} \%$ era ridotta (valore $<38 \%$ ) in una percentuale molto elevata di pazienti, sia tra quelli considerati normali $(78 \%)$, sia malnutriti $(100 \%)$. Tale risultato sembra avere particolare rilevanza se si considera che BMI era ridotto soltanto nel $10 \%$ dei pazienti.

Nella Tabella I sono riportate le correlazioni significative ottenute. Non sono state registrate correlazioni significative tra l'età dialitica e i vari parametri nutrizionali ottenuti sia mediante esame "standard" sia impedenziometrico. Si sottolineano invece le correlazioni significative registrate tra l'età dei pazienti e alcuni dei parametri derivati dal BIA (Xc, PA, $\mathrm{BCM} \%, \mathrm{ECW} \%, \mathrm{ECM} / \mathrm{BCM}) \mathrm{e}$ quelle rilevate tra $\mathrm{AI}$ e TBW\%, FFM\%, FM\%. Si segnalano inoltre (dati non riportati in tabella) le correlazioni significative $(\mathrm{p}<.001)$ osservate tra FM\% e alcuni indici antropometrici derivati dall'esame "standard": BMI $(\mathrm{r}=.51)$, \%IBW $(\mathrm{r}=$ $.60)$, TST $(r=.68)$, \%BF $(r=.61)$. Non è stata registrata alcuna correlazione tra i valori di MAMC e i parametri bioimpedenziometrici.

Al termine del periodo di follow-up durato 48 mesi, durante i quali sono stati seguiti 87 dei 134 pazienti oggetto del nostro studio, la mortalità, risultata del $22 \%$, era significativamente $(\mathrm{p}<.01)$ più alta fra $\mathrm{i}$ pazienti malnutriti rispetto a quelli normali (Fig. 5). Tra i numerosi parametri nutrizionali considerati quelli che si sono correlati significativamente sono stati PRE $(\mathrm{r}=.511 \mathrm{p}<.001)$ e PA $(\mathrm{r}=$ $.48, \mathrm{p}<.001)$. 
Fig. 1 - Valori medi di Rc, Xc, PA e TBW\% rilevati nei 134 pazienti studiati suddivisi in normali e malnutriti sulla base dell'esame nutrizionale "standard".
Fig. 2 - Valori medi di $B C M \%, E C W \% e$ ECM/BCM rilevati nei 134 pazienti studiati suddivisi in normali $e$ malnutriti sulla base dell'esame nutrizionale "standard".

\section{Rc}

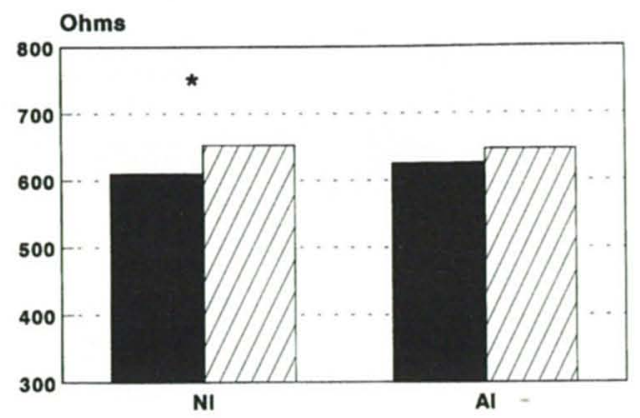

Xc

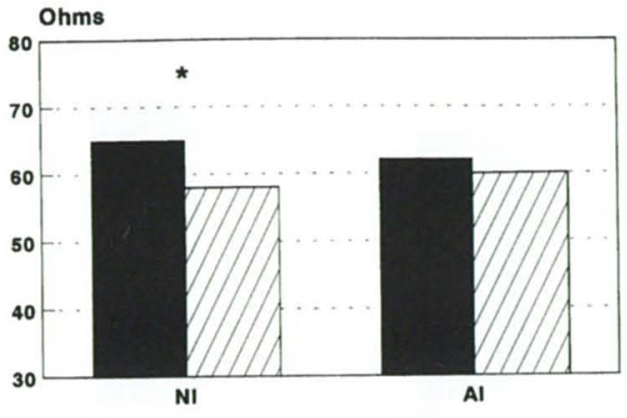

${ }^{*} p<.02$

${ }^{\star} p<.02$

$\mathrm{NI}>85 \% \quad \mathrm{NI}<85 \%$

PA

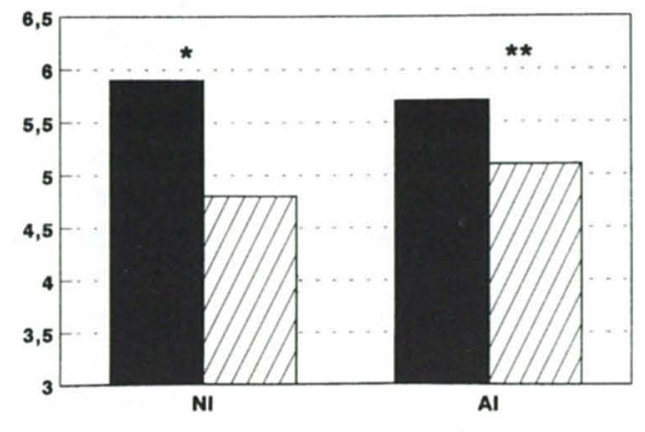

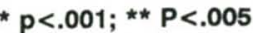

TBW\%

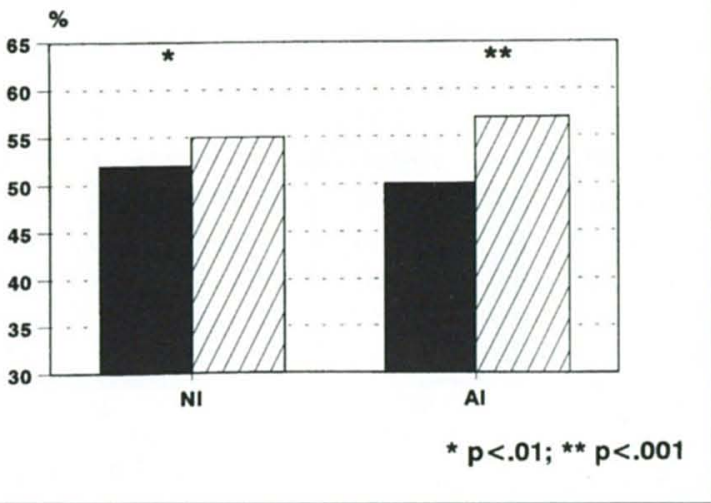

\section{ECW\%}

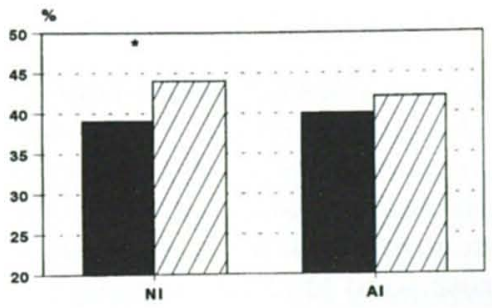

${ }^{*} p<.01$

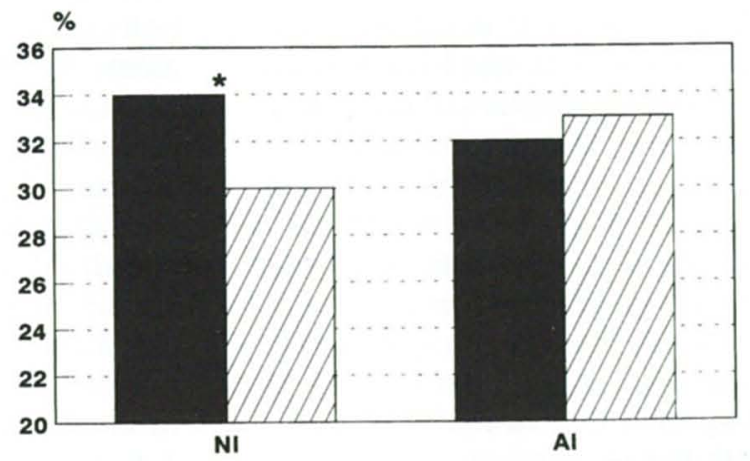

${ }^{*} p<.001$

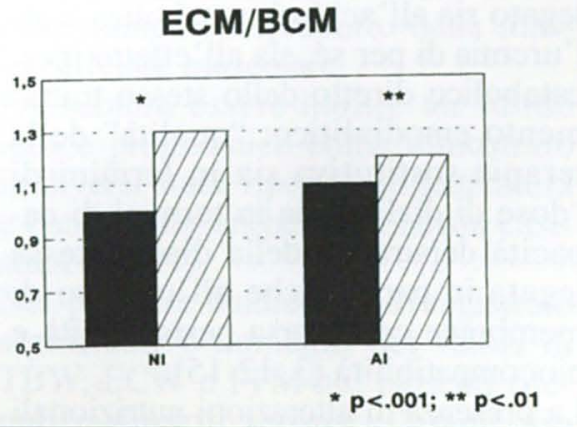




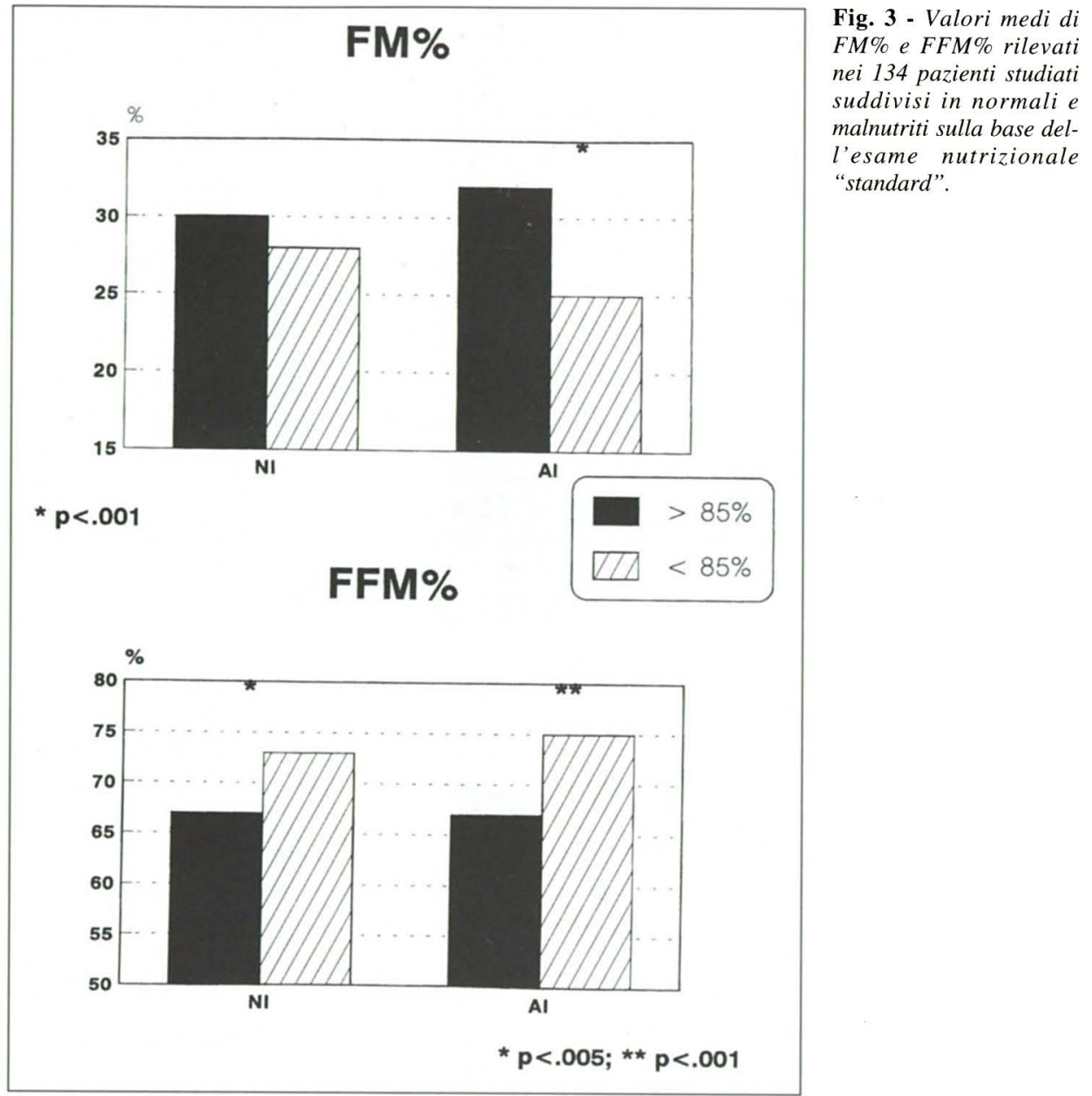

\section{Discussione}

I risultati del presente studio confermano che alterazioni dello stato nutrizionale sono presenti in una elevata percentuale di pazienti con IRC in trattamento emodialitico periodico. Numerose sono le cause di malnutrizione in questi soggetti: riduzione dell'apporto calorico e proteico; alterazioni ormonali (resistenza tessutale all'insulina, iperglucagonemia, iperparatirodismo); stato ipercatabolico legato sia all'acidosi metabolica e all'uremia di per sé, sia all'effetto ipercatabolico diretto dello stesso trattamento emodialitico; "qualità" della terapia sostitutiva sia in termini di "dose di dialisi" sia in termini di capacità depurativa della dialisi stessa legata in parte anche all'utilizzo di membrane con scarsa permeabilità e biocompatibilità $(3,12-15)$.

La presenza di alterazioni nutrizionali sembra avere particolare rilevanza clinica nei pazienti emodializzati. È stato infatti più volte segnalato in letteratura che la malutrizione costituisce uno dei principali fattori prognostici in questi pazienti, aumentandone la morbilità e la mortalità $(6,14,16$, 17). D'altra parte è necessario comunque considerare che l'età avanzata, il tipo di nefropatia causa di IRC, la presenza di malattie croniche concomitanti, la frequente insorgenza di sono di per sé essere causa di malnutrizione e condizionare in misura significativa la prognosi di questi soggetti. Tuttavia, proprio per l'influenza così negativa che la malnutrizione, anche di per sé, sembra avere sul decorso clinico e la prognosi dei pazienti emodializzati è necessario identificare, tra i vari parametri comunemente utilizzati, quelli dotati di maggiore semplicità, sensibilità, specificità, riproducibilità, così da poter essere uticomplicanze acute intercorrenti pos- lizzati anche quali indici prognostici. La valutazione "standard" dello stato nutrizionale in alcuni casi può risultare scarsamente attendibile e sottostimare, o talora sovrastimare, la prevalenza di malnutrizione in questi soggetti. Soprattutto a causa del sovraccarico idrico i parametri antropometrici, in particolare BMI, possono essere sovrastimati mentre al contrario le proteine sieriche, a causa dell'emodiluizione, possono risultare falsamente sottostimate. Tra i diversi parametri "standard" quello che sembra avere maggiore significato, anche dal punto di vista prognostico, è l'albumina (18). In uno studio condotto da Lowrie E.G. et al. In una vasta popolazione di emodializzati, è stato chiaramente dimostrato che il rischio di morte era sette volte più alto nei pazienti con valori di albuminemia inferiori a $30 \mathrm{~g} / \mathrm{L}$. Questo dato è stato più volte confermato anche in altri studi riportati in letteratura $(14,19)$. Va tuttavia considerato che molte delle malattie croniche concomitanti così frequenti in questi pazienti, soprattutto a carico dell'apparato cardiovascolare e gastrointestinale, possono di per sé modificare i vari parametri utilizzati per la valutazione dello stato nutrizionale e limitarne quindi il loro significato clinico e prognostico. Anche la transferrina, utilizata in passato quale valido indice nutrizionale, ha perso oggi molto del suo valore in questo senso dal momento che viene principalmente influenzata dalle continue fluttuazioni del metabolismo del ferro, soprattutto nei pazienti trattati con Eritropoietina. Recentemente il IGF-1 (Insulin-like Grow Factor 1) è stato proposto quale uno dei più precoci e sensibili indici di malnutrizione nei pazienti emodializzati $(3,20)$. Al momento attuale, tuttavia, l'impiego di questo parametro nella pratica clinica risulta scarsamente proponibile.

In un nostro precedente studio, condotto su 91 pazienti emodializzati, i parametri nutrizionali più sensibili, in quanto correlati con il maggior numero di complicanze cliniche, risultavano \%BF, MAMC, PRE e TLC. In particolare, PRE si correlava in misura indipendente con la sopravvivenza 


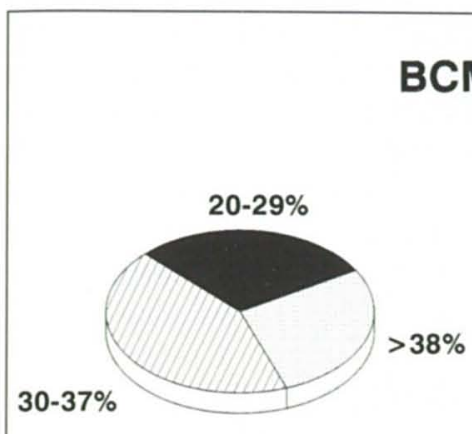

NORMALI

(NI > 85\%)

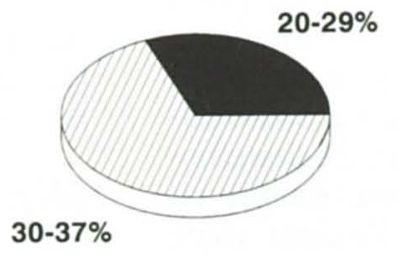

MALNUTRITI

(NI $<85 \%)$

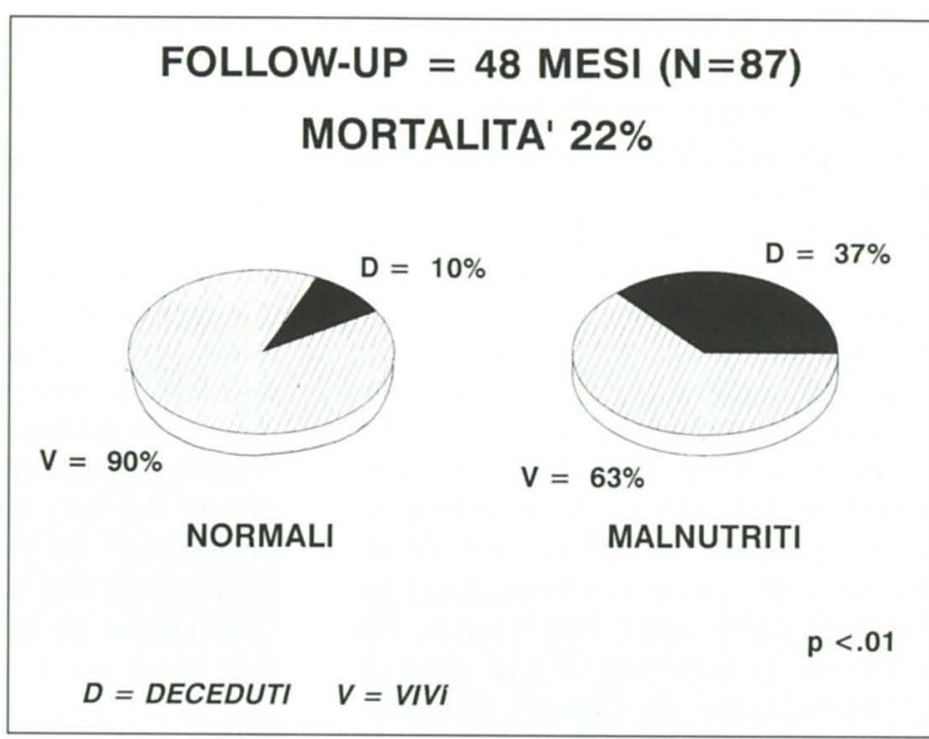

Fig. 5 - Mortalità di 87 dei 134 pazienti studialti seguiti per un periodo di 48 mesi.

Fig. 4 - Distribuzione percentuale di BCM\% nei 134 pazienti studiati suddivisi in normali e malnutriti sulla base dell'esame nutrizionale "standard". Valore normale $>38 \%$; riduzione moderata 30-37\%; riduzione grave $20-29 \%$.

dei pazienti seguiti per un periodo di 72 mesi (16). Anche nel presente studio la prealbumina si è correlata significativamente $(\mathrm{r}=.51, \mathrm{p}<.001)$ con la sopravvivenza dei pazienti seguiti per 48 mesi.

L'esame impedenziometrico è stato largamente impiegato per la valutazione della composizione corporea e quindi dello stato nutrizionale dei pazienti in trattamento emodialitico. BIA infatti, attraverso la misura del contenuto totale di acqua corporea, consente di rilevare indirettamente la percentuale di massa grassa, di quella priva di grasso e della massa cellulare (21-23). Questa tecnica pertanto può essere utilizzata sia nella valutazione dello stato nutrizionale sia dello stato di idratazione del paziente consentendo anche di monitorare le variazioni dei liquidi corporei durante la seduta dialitica. BIA si basa sul principio bioelettrico dell'impedenza che esprime la resistenza di un circuito, in questo caso il corpo umano, attraversato da corrente elettrica alternata. Questa resistenza (Rc) risulta inversamente correlata al contenuto idrico dell'organismo. Altra porzione dell'impedenza è la reattanza $(\mathrm{Xc})$, indice dell'entità delle proprietà "capacitive" degli elementi quali le membrane cellulari $(21,24)$.

In passato BIA è stato maggiormente utilizzato per la valutazione dello sta- to di idratazione dei pazienti emodializzati per stabilirne con maggiore accuratezza quello che viene definito "peso secco". In realtà studi recenti hanno convalidato il valore di questa metodica, pur se con qualche limite, nello studio dello stato nutrizionale di questi soggetti. In particolare è stata dimostrata una correlazione altamente significativa $(r=.92)$ tra il valore di massa cellulare ottenuto mediante BIA e quello derivato attraverso altre metodiche più sofisticate $(25,26)$.

Anche sulla base dei risultati ottenuti nel presente studio, le indicazioni fornite dall'esame BIA sullo stato nutrizionale sembrano essere diverse e complementari rispetto a quelle ottenute dall'esame nutrizionale "standard". Tra i parametri derivati dal BIA quelli che sembrano essere $i$ più specifici e sensibili sono BCM\%, PA e EC/BC.

Riguardo a $\mathrm{BCM} \%$, questa è risultata ridotta in una percentuale molto elevata di pazienti, anche fra quelli considerati normali secondo l'esame nutrizionale "standard". Ciò sta ad indicare che nei pazienti emodializzati può esistere una considerevole compromissione della massa muscolare non altrimenti evidenziabile utilizzando i più comuni parametri nutrizionali. Anche PA sembra essere un indice nutrizionale particolarmente sensibile, affidabile e riproducibile nei pazienti emodializzati. PA, infatti, attraverso le alterazioni elettriche legate alle membrane cellulari associate a diversa ripartizione dei compartimenti intra- ed extra-cellulari, riflette in misura estremamente sensibile le modificazioni dello stato nutrizionale nonché del contenuto dei liquidi corporei. Anche nel presente studio PA è risultato significativamente più basso nei pazienti malnutriti. Questo parametro sembra essere particolarmente influenzato dall'età dei pazienti studiati, come dimostrato anche dall'elevata correlazione esistente tra $\mathrm{i}$ due parametri $(\mathrm{r}=-0.66$, $\mathrm{p}<.001$ ). Questo dato è una ulteriore conferma dell'attendibilità di PA nella valutazione della composizione corporea tenuto conto proprio del fatto che, tra le varie cause di malnutrizione negli emodializzati, l'età avanzata è una fra quelle di maggiore rilievo essendo di per sé causa di depauperamento soprattutto della massa cellulare muscolare.

PA sembra essere inoltre un valido indice prognostico come dimostrato in diversi studi riportati in letteratura e confermato anche nella nostra casistica $(24,27)$.

Nel presente studio è stato registrato significativo aumento dei valori di TBW, ECW e FFM nei pazienti ritenuti malnutriti secondo l'esame nu- 
trizionale "standard". Ciò non dovrebbe essere considerato indice di effettiva iperidratazione bensì segno indiretto dell'aumento relativo della quota idrica legato alla stessa riduzione della massa cellulare. Negli stati di malnutrizione, infatti, la quota di massa cellulare "erosa" viene sostituita da fluidi extracellulari il cui aumento percentuale è solo relativo e non assoluto. In questi casi i fluidi corporei non possono essere considerati in eccesso tali da dover essere rimossi mediante ultrafiltrazione. D'altra parte nei pazienti emodializati risulta di particolare importanza sia verificare la presenza di uno stato di iperidratazione sia rilevare precocemente i segni di malnutrizione. Il rapporto ECM/BCM derivato dal BIA a tale scopo può fornire utili informazioni. Nel presente studio i valori di massa priva di grasso (FFM) sono risultati paradossalmente più alti nei pazienti considerati malnutriti. Va peraltro tenuto in considerazione che in questi soggetti la massa magra non deve essere considerata come un unico compartimento, ma deve essere valutata in due masse distinte: massa cellulare, espressa da BCM e massa extracellulare idrica, espressa da ECW. Nei soggetti normali, vale a dire con normale massa cellulare e normale stato di idratazione, il rapporto ECM/BCM corrisponde circa ad 1. In condizioni patologiche, sia caratterizzate da iperidratazione sia da malnutrizione, questo rapporto in entrambi i casi risulta $>1$. Negli stati di sovraccarico idrico, senza malnutrizione, tuttavia, il valore di BCM risulta normale e l'aumento di $\mathrm{ECM} / \mathrm{BCM}$ riflette un reale aumento dei liquidi corporei. Al contrario negli stati di malnutrizione il rapporto $\mathrm{ECM} / \mathrm{BCM}$ risulta ugualmente $>1$ ma in questo caso per riduzione del valore di BCM. L'aumento di ECM deve essere considerato solo relativo e non rappresenta un reale sovraccarico idrico.

Riguardo alla valutazione della massa grassa, nel presente studio è stata confermata la buona correlazione esistente tra i parametri ottenuti mediante le misure antropometriche e l'esame BIA. Deve essere considera- to, tuttavia, che la stima della massa grassa con metodo BIA può risultare talora inaffidabile, soprattutto nei soggetti con variazione del contenuto idrico dell'organismo, quali proprio i soggetti emodializzati. In particolare, se la misura viene eseguita in questi pazienti prima della seduta emodialitica la massa grassa può risultare notevolmente sottostimata, mentre risulta sovrastimata se l'esame viene effettuato immediatamente dopo la dialisi. In uno studio recentemente pubblicato da Oe B. et al. è stato confermato che negli emodializati la valutazione della massa grassa ottenuta mediante BIA risente in misura maggiore, rispetto alla plicometria, delle variazioni dei liquidi corporei (23). Tale limite deve essere tenuto in considerazione per una corretta interpretazione dei dati ottenuti, considerando soprattutto il momento in cui l'esame BIA viene effettuato rispetto alla seduta emodialitica. Nel presente studio la valutazione impedenziometrica è stata eseguita $30^{\prime}$ dal termine del trattamento dialitico, tempo ritenuto sufficiente per ottenere uno stato di equilibrio dei liquidi corporei e quindi dei risultati attendibili. $\mathrm{Ri}$ guardo all'esame BIA, va comunque tenuta in considerazione la maggiore riproducubilità di questa tecnica nella valutazione della composizione corporea rispetto alle misure antropometriche che troppo possono risentire della variabilità legata all'operatore.

Dai risultati ottenuti nel presente studio possiamo concludere che l'esame BIA rappresenta un valido metodo nella valutazione dello stato nutrizionale dei pazienti emodializati, in quanto semplice, sensibile, affidabile e altamente riproducibile. In particolare sembra fornire informazioni diverse, e al tempo stesso complementari, rispetto a quelle ottenute mediante esame nutrizionale "standard" potendo evidenziare uno stato di malnutrizione talora non altrimenti dimostrabile. A tale scopo risultano particolarmente sensibili gli indici di massa corporea derivati dal BIA in rapporto allo stato di idratazione: BCM\%. PA, ECM/BCM.

Studi longitudinali sono necessari per valutare se l'esame BIA si dimostri realmente utile e sensibile nella "sorveglianza" nutrizionale dei pazienti emodializati consentendo di identificare precocemente quelli a maggiore rischio di malnutrizione e quindi di una più alta morbilità e mortalità.

\section{Riassunto}

Scopo dello studio è valutare lo stato nutrizionale di 134 pazienti ED clinicamente stabili $(83 \mathrm{M}, 51 \mathrm{~F}$, età media $62 \pm 14$ aa) mediante esame nutrizionale "standard" (indici antropometrici, bioumorali, immunitari) e mediante esame impedenziometrico. I pazienti sono stati seguiti per 48 mesi.

Sulla base dei parametri nutrizionali "standard" i pazienti sono sati suddivisi in Normali $(\mathrm{N}=59 \%)$ e Malnutriti $(M=41 \%)$.

I parametri indicativi della massa grassa ottenuti mediante esame antropometrico e valutazione impedenziometrica si sono correlati significativamente $(\mathrm{p}<.001)$. Una correlazione inversa significativa $(\mathrm{p}<.001)$ è stata rilevata tra FFM\% e gli indici antropometrici. $\mathrm{BCM} \%$ è risultata ridotta in una alta percentuale di pazienti ( $\mathrm{N}$ $78 \%$, M 100\%). FFM\% e TBW\% si sono correlati inversamente con gli indici antropometrici $(\mathrm{p}<.01)$. Durante il periodo di follow-up la mortalità è stata del $22 \%$, significativamente più alta nei pazienti $\mathrm{M}(\mathrm{p}<.01)$. I parametri significativamente correlati con la sopravvivenza $(\mathrm{p}<.001)$ erano prealbumina e PA.

I risultati del presente studio indicano che: 1) l'aumento di FFM\% osservato negli $\mathrm{M}$ potrebbe essere considerato un segno indiretto di aumento dei liquidi corporei per riduzione di BCM; 2) l'esame bio impedenziometrico fornisce ulteriori informazioni da quelle ottenute con l'esame standard; 3) la significativa riduzione di $\mathrm{BCM}$ con normalità di BMI e FM\% è fortemente indicativa di perdita di massa muscolare e di deplezione proteica.

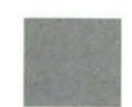




\section{BIBLIOGRAFIA}

1. Hakim RM, Levin N. Malnutrition in hemodialysis patients. Am J Kidney Dis 1993; 21: 125-37.

2. Ikizler TA, Hakim RM. Nutrition in end-stage renal disease. Kidney Int 1996; 50: 343-57.

3. Qureshi AR, Alvestrand A, Danielsson A et al. Factors predicting malnutrition in hemodialysis patients: a cross-sectional study. Kidney Int 1998; 53: 773-82.

4. Bergstrom J. Why are dialysis patients malnourished? Am J Kidney Dis 1995; 26: 229-41.

5. Acchiardo SR, Moore LW. Malnutrition as the main factor in morbidity and mortality of hemodialysis patients. Kidney Int 1993; 24: 199-203.

6. Marckmann P. Nutritional status and mortality of patients in regular dialysis therapy. J Intern Med 1989; 226: 429.

7. Kopple JD. Effect of nutrition on morbidity and mortality in maintenance dialysis patients. Am J Kidney Dis 1994; 24: 1002-9.

8. Mandolfo S, Imbascati E. Bioimpedenziometria, antropometria e cinetica: confronto e ripetibiltià. Giorn It Nefrol 1993; 10: 111-5.

9. Di lorio B, Talluri T, Terracciano V, Altieri C. Valutazione della composizione corporea mediante indici derivati dalla bioimpedenziometria. Giorn It Nefrol 1996; 13: 101-7.

10. Chertow GM, Lazarus JM, Lew NL, Ma L, Lowrie EG. Bioimpedance norms for the hemodialysis population. Kidney Int 1997; 52: 1617-21.

11. Durnin JVCA, Womersley J. Body fat assessed from total body density and its estimation from skinfold thikness measurements on 481 men and women aged from 16 to
72 years. Br J Nutr 1974; 32: 77-81.

12. Hakim RM. Clinical implications of hemodialysis membrane biocompatibility. Kidney Int 1993: 44: 484-94.

13. Parker TF. Role of dialysis dose on morbidity and mortality in maintenance hemodialysis patients. Am J Kidney Dis 1994; 24: 981-9.

14. Owen WF, Lew NL, Liu Y, Lowrie EG, Lazarus JM. The urea reduction ratio and serum albumin concentration as predictor of mortality in patients undergoing hemodialysis. N Engl J Med 1993; 329: 1001-6.

15. Parker III TF, Wingard RL, Husni L, Ikizler TA, Parker RA, Hakim RM. Effect of the membrane biocompatibility on nutritional parameters in chronical hemodialysis patients. Kidney Int 1996; 49: 551-6.

16. Chiappini MG, Selvaggi G, Traietti P, Bartoli R. Nutritional status in hemodialysis (HD) patients (PTS): a cross-sectional and longitudinal study. Current Ther Nephrol 1993; p 337-43.

17. Lazarus JM, Owen WF. Role of bioincompatibility in dialysis morbidity and mortality. Am J Kidney Dis 1994; 24: 1019-32.

18. Lowrie EG, Huang WH, Lew NL. Death risk predictors among peritoneal dialysis and hemodialysis patients: a preliminary comparison. Am J Kidney Dis 1995; 26: 220-8.

19. Iseki K et al. Serum albumin is a strong predictor of death in cronic dialysis patients. Kidney Int 1994; 44: 115-9.

20. Sanaka T, Shinobe M, Ando M et al. IGF-I as an early indicator of malnutrition in patients with endstage renal disease. Nephron 1994; 67: 73-81.

21. Piccoli A, Rossi B, Pillon L, Bucciante G. A new method for monitoring body fluid variation by bioimpedance analysis: the Rxc graph. Kidney Int 1994; 44: 534-9.
22. Madore F, Wuest M, Ethier JH. Nutritional evaluation of hemodialysis patients using an impedance index. Clin Nephrol 1994; 41: $377-$ 82.

23. Oe B, De Fijter CWH, Oe PL, Stevens P, De Vries PMJM. Four-site skinfold anthropometry (FSA) versus body impedance analysis (BIA) in assessing nutritional status of patients on maintenance hemodialysis: wich method is to be preferred in routine patient care? Clin Nephrol 1998; 49: 180-5.

24. Maggiore Q, Nigrelli S, Ciccarelli $\mathrm{C}$ et al. Nutritional and prognostic correlates of bioimpedance indexes in hemodialysis patients. Kidney Int 1993; 50: 2103-8.

25. Chertow GM, Lowrie EG, Wilmore DW et al. Nutritional assessment with bioeletrical impedance analysis in maintenance hemodialysis patients. J Am Soc Nephrol 1995; 6: 75-81.

26. Abrahamsen B, Hansen TB, Hogsberg IM, Pederson FB, BeckNielsen H. Impact of hemodialysis on dual X-ray absorptiometry, bioelectrical impedance measurements, and anthropometry. Am J Clin Nutr 1996; 63: 80-6.

27. Ott $\mathrm{M}$, Fischer $\mathrm{H}$, Polat $\mathrm{H}$ et al. Bioelectric impedance analysis as a predictor of survival in patients with HIV infections. J of Acquired Immune Deficiency Syndromes and Human Retrovirus 1995; 9: 20-4. 